UNIVERSITY OF CALIFORNIA

Agriculture and Natural Resources

LUCIA KAISER, UC Cooperative Extension Nutrition Specialist Emeritus, Department of Nutrition, University of California, Davis; ALBERTO AGUILERA, Lecturer, School of Social Sciences, Humanities, and Arts, University of California, Merced;

\section{JUDITH SILVA, Mental Health} Clinician, Madera County Behavioral Health Services, Madera, California; MARCEL HOROWITZ, Healthy Youth, Families, and Communities Advisor, University of California Cooperative Extension, Yolo County; CATHERINE LAMP, Nutrition, Family, and Consumer Sciences Advisor Emeritus, University of California Cooperative Extension, Tulare County;

MARGARET JOHNS, Nutrition, Family, and Consumer Sciences Advisor Emeritus, University of California Cooperative Extension, Kern County;

DORINA ESPINOZA, Youth, Families, and Communities Advisor, University of California Cooperative Extension, Humboldt County; MICHELE BYRNES, Community Education Specialist 4, CalFresh Healthy Living, University of California, Davis, California; LENNA ONTAI, UCCE Family and Early Childhood Specialist, Department of Human and Community Development, University of California, Davis; AND

KARINA DIAZ RIOS, UCCE

Specialist in Nutrition, University of California, Merced

\section{Part Five: Visual Aids}

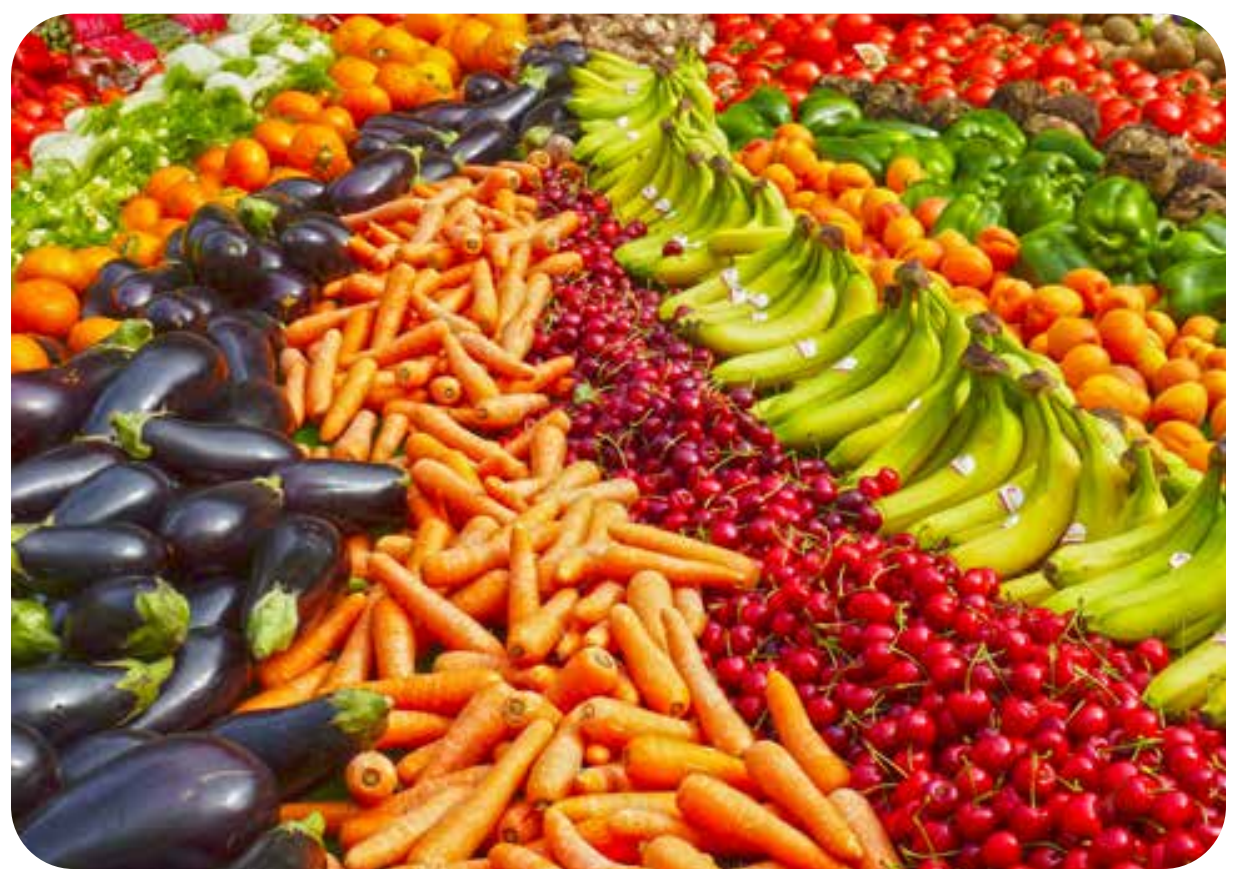

Niños Sanos, Familia Sana (Healthy Children, Healthy Family) is a nutrition education curriculum, designed for Latino parents with young children. The goal is to improve nutrition and physical activity to prevent childhood obesity and promote health. This publication includes visual aids that can be used with the 20 lesson plans available in publication 8583. Instructions on how to use these visuals with the lesson are provided in that publication. 


\section{Nutritional Facts}

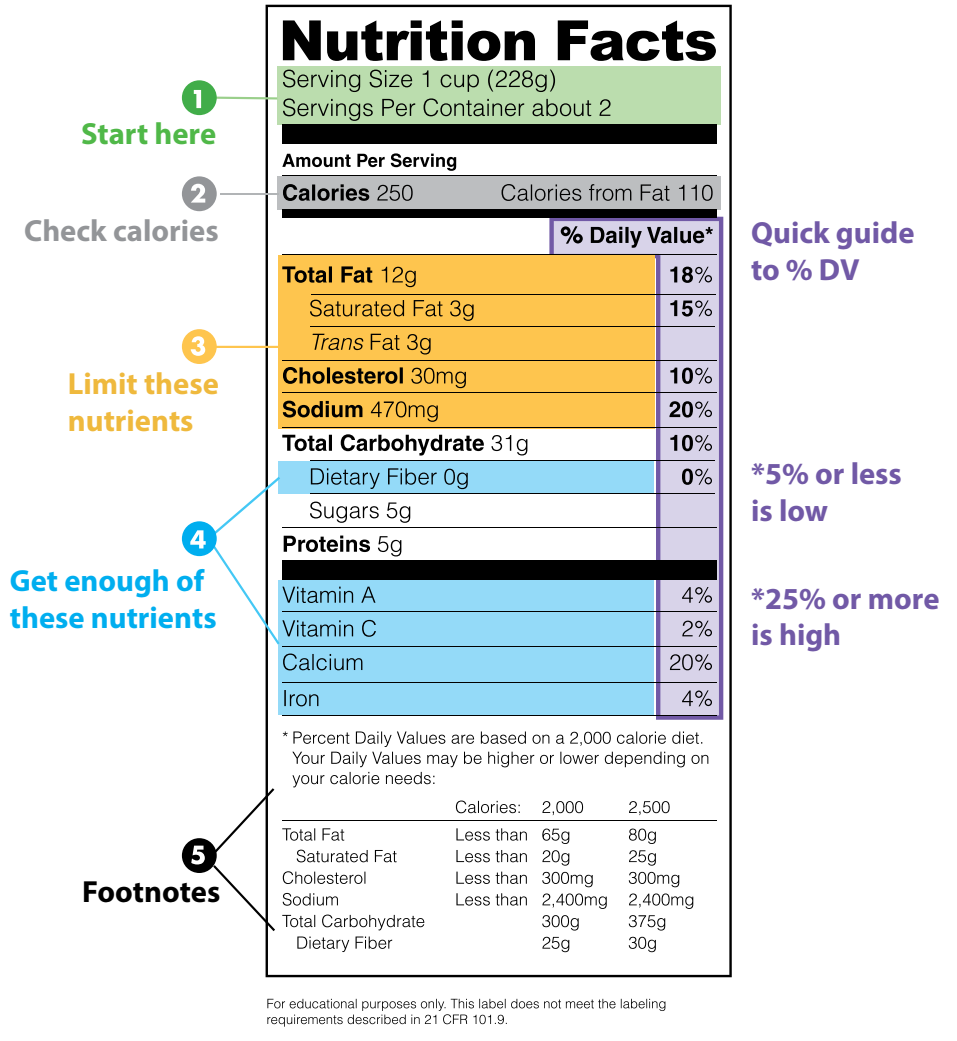

\begin{tabular}{|c|c|c|c|}
\hline \multicolumn{4}{|c|}{ Datos de Nutrición } \\
\hline \multicolumn{4}{|c|}{ Cantidad por Ración } \\
\hline \multirow[t]{2}{*}{ Calorías 250} & \multicolumn{3}{|c|}{ Calorías de grasa 110} \\
\hline & \multicolumn{3}{|c|}{ \% Valor diario } \\
\hline \multicolumn{3}{|l|}{ Grasa Total $12 \mathrm{~g}$} & $18 \%$ \\
\hline \multicolumn{3}{|c|}{ Grasa Saturada $3 \mathrm{~g}$} & $15 \%$ \\
\hline \multicolumn{4}{|c|}{ Grasa Trans $3 \mathrm{~g}$} \\
\hline \multicolumn{3}{|c|}{ Colesterol $30 \mathrm{mg}$} & $10 \%$ \\
\hline \multicolumn{3}{|l|}{ Sodio $470 \mathrm{mg}$} & $20 \%$ \\
\hline \multicolumn{3}{|c|}{ Carbohidrato Total $31 \mathrm{~g}$} & $10 \%$ \\
\hline \multicolumn{3}{|c|}{ Fibra Dietética 0g } & $0 \%$ \\
\hline \multicolumn{4}{|l|}{ Azúcares $5 \mathrm{~g}$} \\
\hline \multicolumn{4}{|l|}{ Proteínas $5 \mathrm{~g}$} \\
\hline \multicolumn{3}{|l|}{ Vitamina A } & $4 \%$ \\
\hline \multicolumn{3}{|l|}{ Vitamina C } & $2 \%$ \\
\hline \multicolumn{3}{|l|}{ Calcio } & $20 \%$ \\
\hline \multicolumn{3}{|l|}{ Hierro } & $4 \%$ \\
\hline \multicolumn{4}{|c|}{$\begin{array}{l}\text { "Los porcentajes de Valores Diarios están basados en } \\
\text { una dieta de } 2,000 \text { calorías. Sus Valores Diarios pueden } \\
\text { ser mayores o menores según sus necesidades } \\
\text { calóricas: }\end{array}$} \\
\hline & Calorias: & 2,000 & 2,500 \\
\hline $\begin{array}{l}\text { Grasas totales } \\
\text { Grasas saturadas } \\
\text { Colesterol } \\
\text { Sodio } \\
\text { Carbohidratos totales } \\
\text { Fibra dietética }\end{array}$ & $\begin{array}{l}\text { Menos de } \\
\text { Menos de } \\
\text { Menos de } \\
\text { Menos de }\end{array}$ & $\begin{array}{l}65 \mathrm{~g} \\
20 \mathrm{~g} \\
300 \mathrm{mg} \\
2,400 \mathrm{mg} \\
300 \mathrm{~g} \\
25 \mathrm{~g}\end{array}$ & $\begin{array}{l}80 \mathrm{~g} \\
25 \mathrm{~g} \\
300 \mathrm{mg} \\
2,400 \mathrm{mg} \\
30 \mathrm{~g} \\
30 \mathrm{~g}\end{array}$ \\
\hline
\end{tabular}

For educational purposes only. This label does not meet the labeling
requirements described in 21 CFR 101.9 . 


\section{Nutritional Facts}

\section{CEREAL A}

(Oatmeal)

\begin{tabular}{|c|c|c|c|}
\hline \multicolumn{4}{|c|}{$\begin{array}{l}\text { Nutrition Facts } \\
\text { Serving Size .5 cup (40g) } \\
\text { Servings Per Container } 30\end{array}$} \\
\hline \multicolumn{4}{|l|}{ Amount Per Serving } \\
\hline \multirow[t]{2}{*}{ Calories 150} & \multicolumn{3}{|c|}{ Calories from Fat 25} \\
\hline & \multicolumn{3}{|c|}{ \% Daily Value ${ }^{\star}$} \\
\hline \multicolumn{2}{|l|}{ Total Fat 3g } & & $5 \%$ \\
\hline \multicolumn{2}{|c|}{ Saturated Fat $0.5 \mathrm{~g}$} & & $3 \%$ \\
\hline \multicolumn{4}{|c|}{ Trans Fat $0 \mathrm{~g}$} \\
\hline \multicolumn{2}{|c|}{ Cholesterol Omg } & & $0 \%$ \\
\hline \multicolumn{2}{|l|}{ Sodium Omg } & & $0 \%$ \\
\hline \multicolumn{2}{|c|}{ Total Carbohydrate 27g } & & $9 \%$ \\
\hline \multicolumn{2}{|c|}{ Dietary Fiber $4 \mathrm{~g}$} & & $16 \%$ \\
\hline \multicolumn{4}{|c|}{ Sugars $1 \mathrm{~g}$} \\
\hline \multicolumn{2}{|l|}{ Proteins 5g } & & $10 \%$ \\
\hline \multicolumn{2}{|l|}{ Iron } & & $10 \%$ \\
\hline \multicolumn{4}{|c|}{$\begin{array}{l}\text { *Percent Daily Values are based on a 2,000 calorie } \\
\text { diet.Your Daily Values may be higher or lower depend- } \\
\text { ing on your calorie needs: }\end{array}$} \\
\hline & Calories: & 2,000 & 2,500 \\
\hline Total Fat & Less than & $65 \mathrm{~g}$ & $80 \mathrm{~g}$ \\
\hline Saturated Fat & Less than & & $25 \mathrm{~g}$ \\
\hline Cholesterol & Less than & $300 \mathrm{mg}$ & $300 \mathrm{mg}$ \\
\hline $\begin{array}{l}\text { Sodium } \\
\text { Total Carbohydrate }\end{array}$ & Less than & $\begin{array}{l}2,400 \mathrm{mg} \\
300 \mathrm{~g}\end{array}$ & $\begin{array}{l}2,400 \mathrm{mg} \\
375 \mathrm{~g}\end{array}$ \\
\hline Dietary Fiber & & & \\
\hline
\end{tabular}

\section{CEREAL B}

(Ready-to-eat, with rasins)

\begin{tabular}{|c|c|c|c|}
\hline \multicolumn{4}{|c|}{$\begin{array}{l}\text { Nutrition Fa } \\
\text { Serving Size } 1 \text { cup } \\
\text { Servings Per Container } 7\end{array}$} \\
\hline \multicolumn{4}{|l|}{ Amount Per Serving } \\
\hline \multirow[t]{2}{*}{ Calories 180} & \multicolumn{3}{|c|}{ Calories from Fat 25} \\
\hline & \multicolumn{3}{|c|}{$\%$ Daily Value ${ }^{\star}$} \\
\hline \multicolumn{2}{|l|}{ Total Fat $1.5 \mathrm{~g}$} & & $2 \%$ \\
\hline \multicolumn{2}{|c|}{ Saturated Fat $0 \mathrm{~g}$} & & $0 \%$ \\
\hline \multicolumn{4}{|c|}{ Trans Fat $0 \mathrm{~g}$} \\
\hline \multicolumn{2}{|l|}{ Cholesterol 0mg } & & $0 \%$ \\
\hline \multicolumn{2}{|c|}{ Potassium $140 \mathrm{mg}$} & & $4 \%$ \\
\hline \multicolumn{2}{|l|}{ Sodium $190 \mathrm{mg}$} & & $8 \%$ \\
\hline \multicolumn{2}{|c|}{ Total Carbohydrate $44 \mathrm{~g}$} & & $15 \%$ \\
\hline \multicolumn{2}{|c|}{ Dietary Fiber $5 \mathrm{~g}$} & & $20 \%$ \\
\hline \multicolumn{4}{|l|}{ Sugars $17 \mathrm{~g}$} \\
\hline \multicolumn{2}{|l|}{ Proteins $4 \mathrm{~g}$} & & $9 \%$ \\
\hline \multicolumn{2}{|l|}{ Vitamin A 10\% } & \multicolumn{2}{|c|}{ Vitamin C 2\% } \\
\hline \multicolumn{2}{|l|}{ Calcium $2 \%$} & \multicolumn{2}{|c|}{ Iron $25 \%$} \\
\hline \multicolumn{4}{|c|}{$\begin{array}{l}\text { "Percent Daily Values are based on a 2,000 calorie } \\
\text { diet.Your Daily Values may be higher or lower depend- } \\
\text { ing on your calorie needs: }\end{array}$} \\
\hline & Calories: & 2,000 & 2,500 \\
\hline & Less than & $65 \mathrm{~g}$ & $80 \mathrm{~g}$ \\
\hline Saturated Fat & Less than & & $25 \mathrm{~g}$ \\
\hline Cholesterol & Less than & $300 \mathrm{mg}$ & $300 \mathrm{mg}$ \\
\hline Sodium & Less than & $2,400 \mathrm{mg}$ & $2,400 \mathrm{mg}$ \\
\hline $\begin{array}{l}\text { Total Carbohydrate } \\
\text { Dietary Fiber }\end{array}$ & & $300 \mathrm{~g}$ & $375 \mathrm{~g}$ \\
\hline & & & \\
\hline
\end{tabular}




\section{LESSON 1.6}

\section{Healthy Eating Visual Aid}

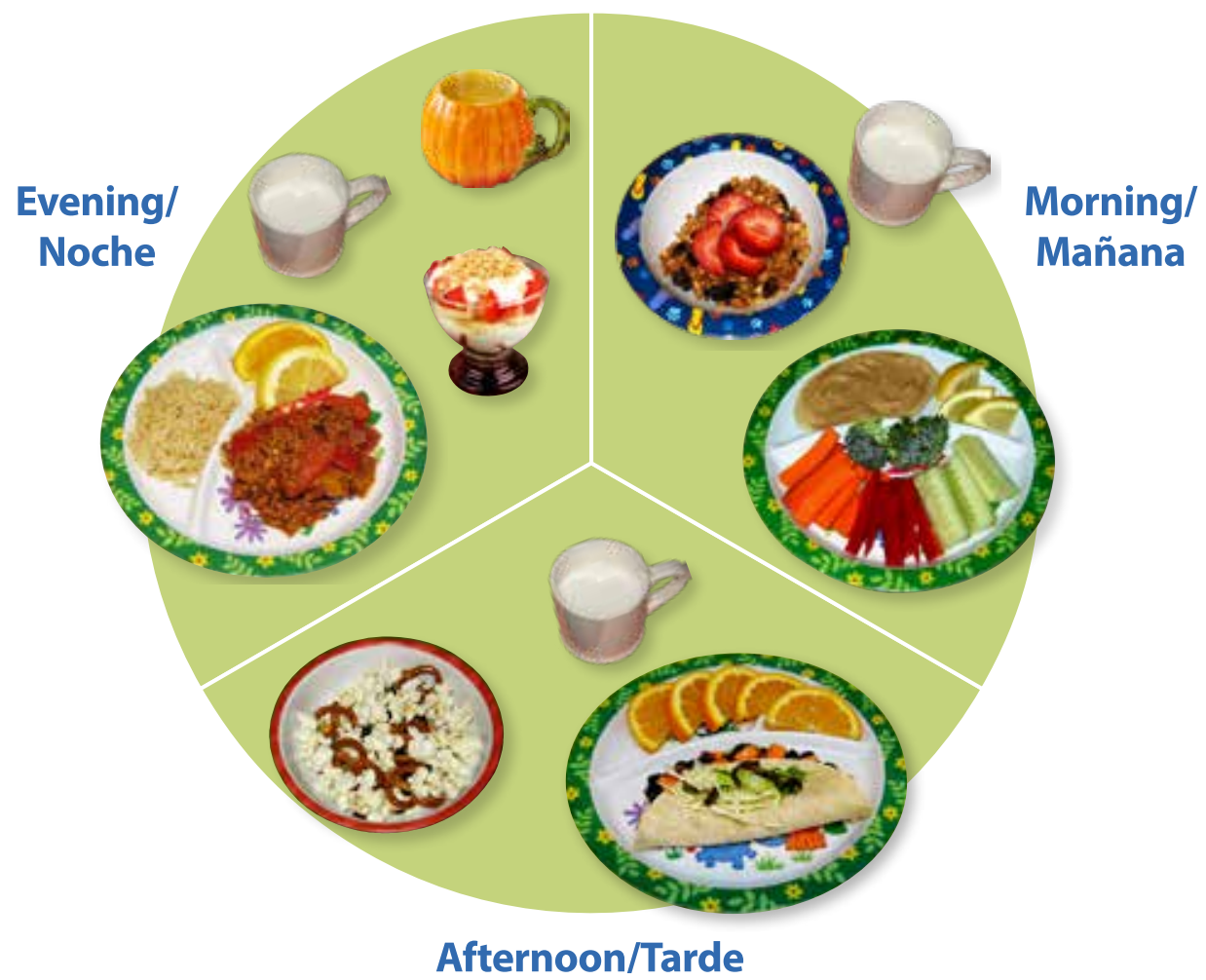

Example of daily healthy food consumption.

\section{Healthy Eating Pattern (I,200 calories)}

\begin{tabular}{|c|c|c|c|c|c|}
\hline Breakfast & $\begin{array}{l}\text { Morning } \\
\text { snack }\end{array}$ & Lunch & $\begin{array}{l}\text { Afternoon } \\
\text { snack }\end{array}$ & Dinner & Bedtime \\
\hline $\begin{array}{l}\text { - } 1 / 4 \text { cup } \\
\text { granola } \\
\text { - } 1 / 2 \text { cup low- } \\
\text { fat } \\
\text { milk } \\
\text { - } 1 / 2 \text { cup } \\
\text { sliced } \\
\text { berries }\end{array}$ & $\begin{array}{l}\cdot 1 / 2 \text { cup } \\
\text { vegetables } \\
\cdot 3 \text { Tbsp } \\
\text { hummus } \\
\cdot \text { water }\end{array}$ & $\begin{array}{l}\cdot 1 \text { southwest } \\
\text { taco ( } 1 \text { corn } \\
\text { tortilla, } 1 / 4 \\
\text { cup beans, } \\
\text { and } 1 / 4 \\
\text { cup sweet } \\
\text { potatoes) } \\
\text {. } 112 \text { orange } \\
\text { - } 112 \text { cup low- } \\
\text { fat milk }\end{array}$ & $\begin{array}{l}\text { - 11/2 cup } \\
\text { popcorn } \\
\text { snack } \\
\text { - water }\end{array}$ & $\begin{array}{l}\cdot 1 / 2 \text { cup } \\
\text { brown rice } \\
\cdot 1 / 2 \text { cup tasty } \\
\text { turkey with } \\
\text { peppers } \\
\cdot 1 / 2 \text { cup low- } \\
\text { fat milk } \\
\cdot 1 / 2 \text { cup fruit } \\
\text { parfait }\end{array}$ & $\begin{array}{l}\text {-1/2 cup } \\
\text { pumpkin } \\
\text { atole }\end{array}$ \\
\hline
\end{tabular}




\section{LESSON 1.6}

\section{Unhealthy Eating Visual Aid}

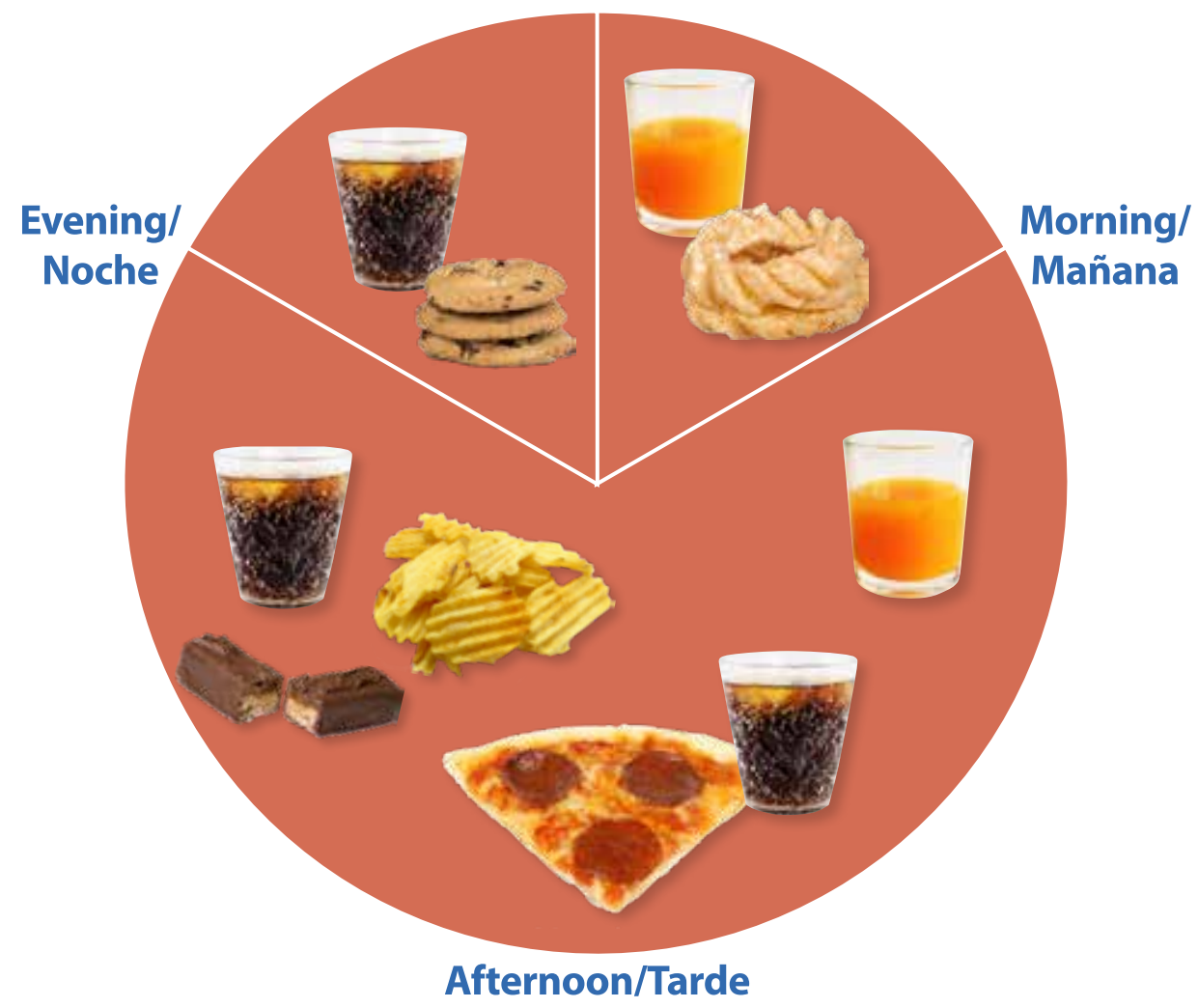

Example of daily unhealthy food consumption.

\section{Unhealthy Eating Pattern (I,232 calories)}

\begin{tabular}{|c|c|c|c|c|c|}
\hline Breakfast & $\begin{array}{l}\text { Morning } \\
\text { snack }\end{array}$ & Lunch & $\begin{array}{l}\text { Afternoon } \\
\text { snack }\end{array}$ & Dinner & Bedtime \\
\hline $\begin{array}{l}\cdot 1 \text { donut } \\
\cdot 3 \text { oz fruit } \\
\text { drink }\end{array}$ & $\begin{array}{l}3 \text { oz fruit } \\
\text { drink }\end{array}$ & $\begin{array}{l}\text { - } 1 \text { slice } \\
\text { pepperoni } \\
\text { pizza } \\
\text { - } 4 \text { oz soda }\end{array}$ & $\begin{array}{l}\text { - candy bar } \\
\text { - bag of chips } \\
\text { - } 4 \text { oz soda }\end{array}$ & . 4 oz soda & $\begin{array}{l}\text { - } 3 \text { chocolate } \\
\text { cookies } \\
\text { - } 4 \text { oz soda }\end{array}$ \\
\hline
\end{tabular}




\section{LESSON 1.7}

\section{Edgar's Day}

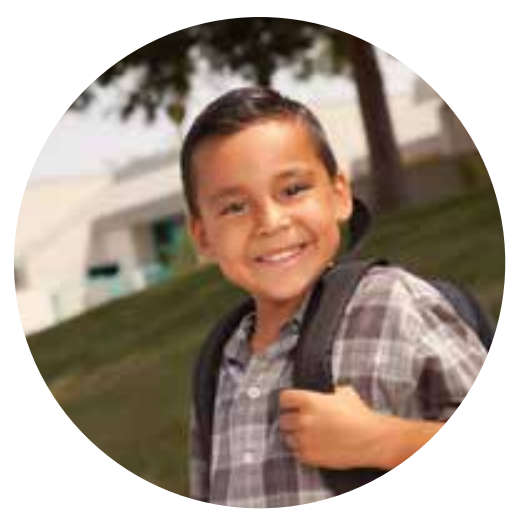

\section{SCHOOL}

\begin{tabular}{|c|c|}
\hline 9:00 & $\begin{array}{l}1 / 2 \text { tortilla } \\
1 \text { teaspoon of refried beans }\end{array}$ \\
\hline 11:30 & $\begin{array}{l}1 / 2 \text { cup salad } \\
1 / 2 \text { orange } \\
1 \text { oz chicken } \\
1 / 4 \text { cup rice } \\
4 \text { oz milk }\end{array}$ \\
\hline
\end{tabular}

\section{HOME}

\begin{tabular}{|c|c|}
\hline $12: 15$ & 3 oz juice \\
\hline $12: 40$ & 3 oz juice \\
\hline 1:00 & 1 hot dog \\
\hline 1:35 & $1 / 2$ cup noodles \\
\hline 2:00 & 2 oz juice \\
\hline 3:10 & $1 / 4$ cup noodles \\
\hline 3:35 & 1 orange \\
\hline 5:00 & 1 oz chicken \\
\hline $6: 00$ & $1 \mathrm{oz}$ sausage \\
\hline \multirow[t]{2}{*}{ 6:30 } & candy \\
\hline & $1 / 2$ pan dulce \\
\hline \multirow[t]{2}{*}{$8: 20$} & $1 / 2$ cup noodles \\
\hline & 3 oz juice \\
\hline
\end{tabular}




\section{Growth Chart Visual Aid}

\section{How Can We Tell if a Child is Gaining a Healthy Amount of Weight?}

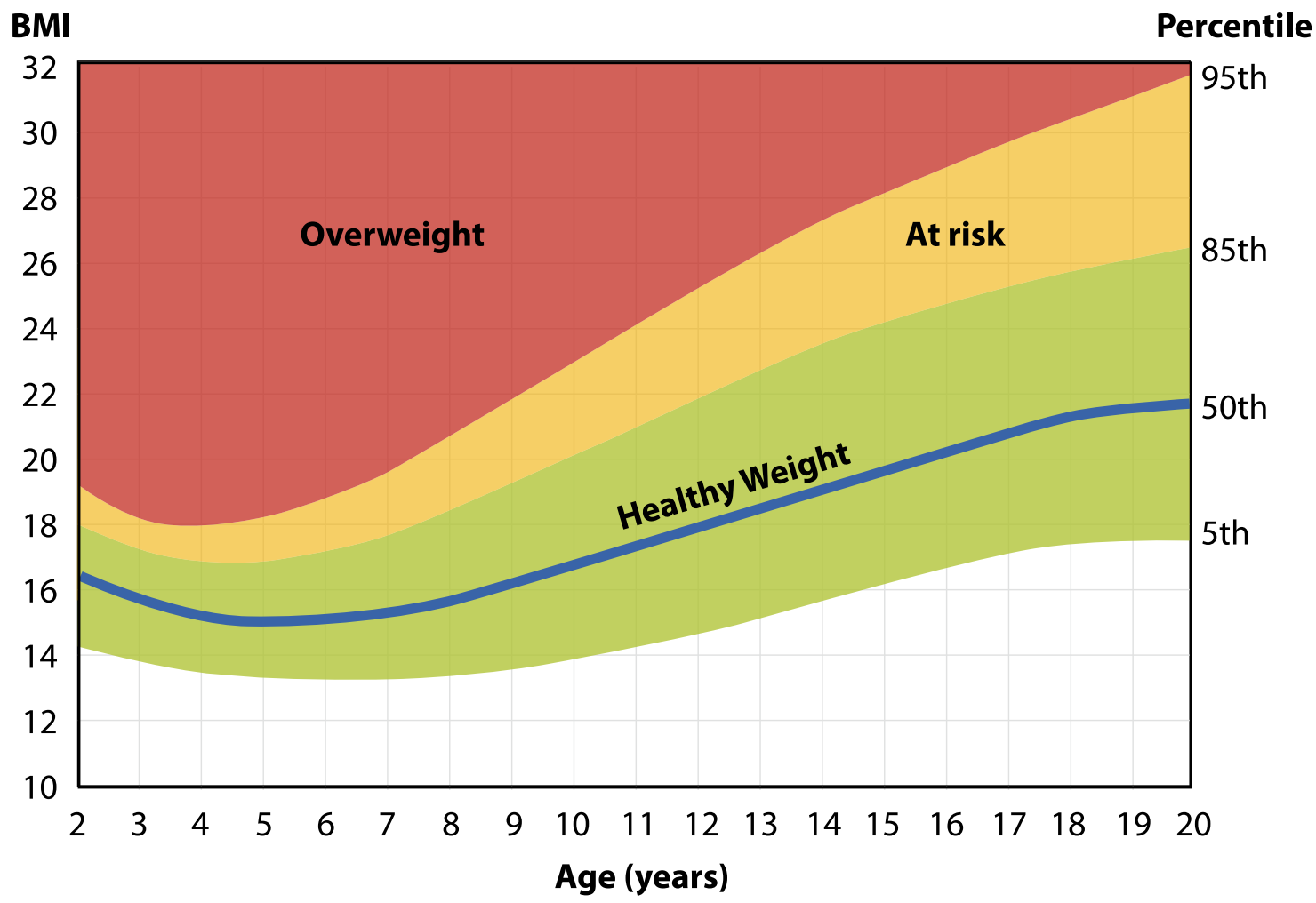

Child growth chart (CDC 2009). 
LESSON 2.1

\section{Compare Three Boys Visual Aid}

\section{Different Children's Weight at 7 Years Old}

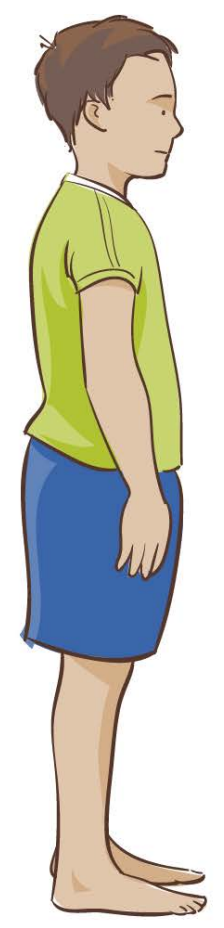

$50 \mathrm{lb}$

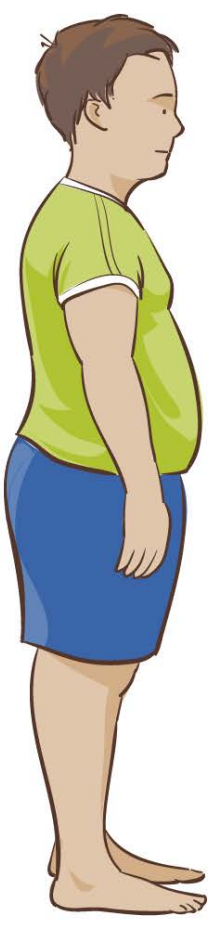

$62 \mathrm{lb}$

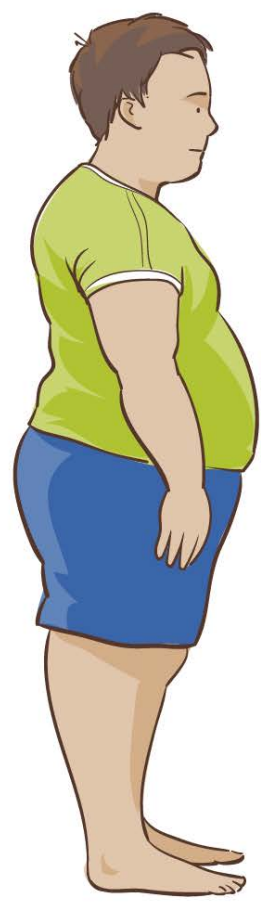

$70 \mathrm{lb}$

\section{Healthy weight}


LESSON 2.1

\section{Ioo Children in a Healthy Community Visual Aid}

\section{In a healthy community of Ioo children, most will be in the green zone, and few will be in the yellow or red zones.}

Healthy Weight



At Risk



Overweight

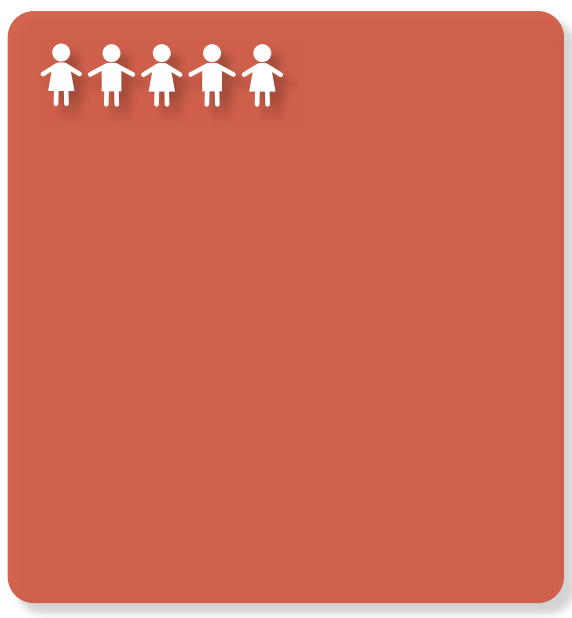

Example of U.S. children's weight status. 
LESSON 2.1

\section{Ioo Children in Our Community Visual Aid}

\section{If we measure children in this community, too many will be in the yellow and red zones.}

\section{"Our Community" Healthy Weight}

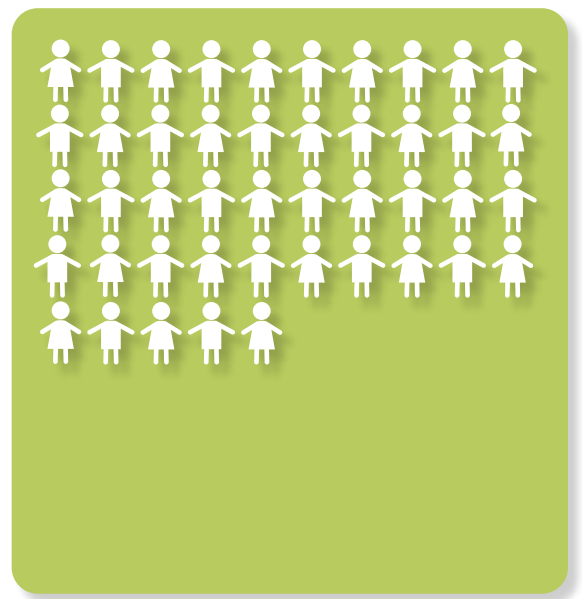
"Our Community"
At Risk

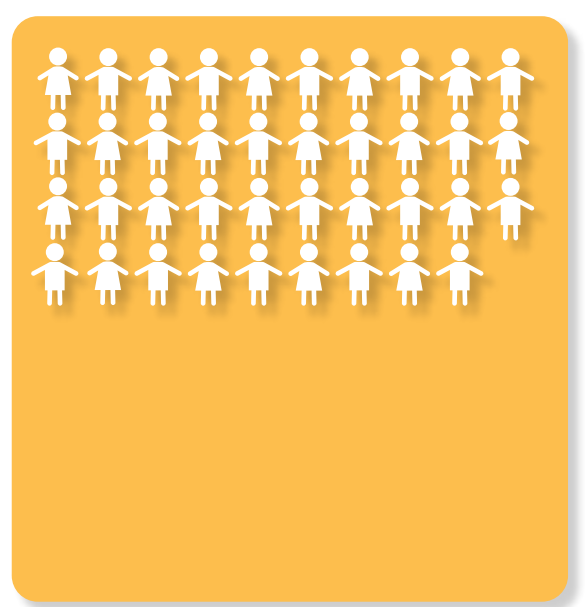

"Our Community" Overweight

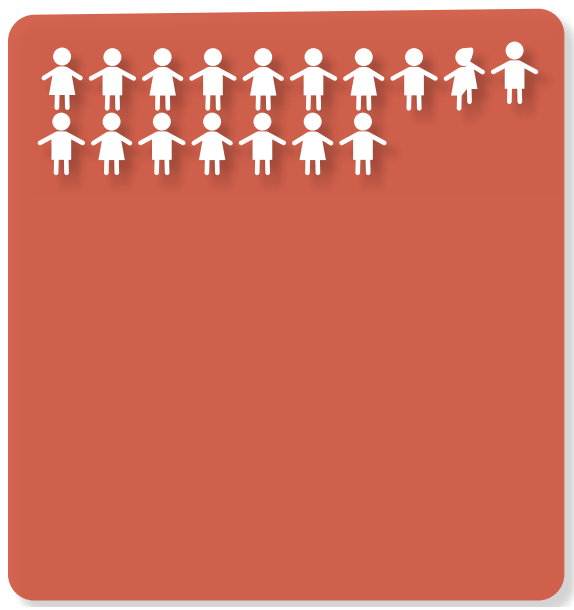

Example of U.S. children's weight status in a local community. 
LESSON 2.2

\section{Motor Skills Visual Aid}


- begins to run

- climbs onto and down from furniture without help

- walks up and down stairs while holding on

- throws ball overhand

- kicks a ball

- stands on tip toes

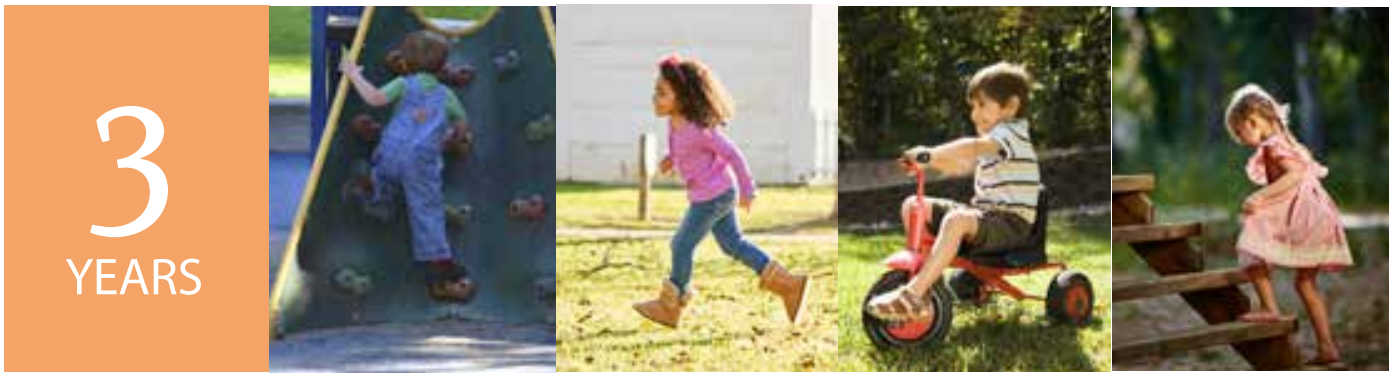

- climbs well

- runs easily

- pedals a tricycle (3-wheel bike)

- walks up and down stairs with one foot on each step
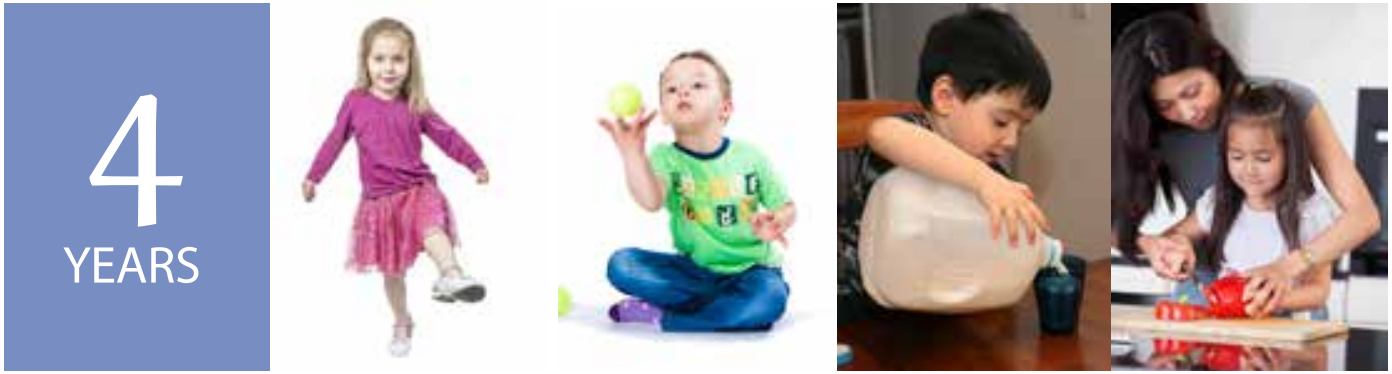

- hops and stands on one foot up to 2 seconds

- catches a bounced ball most of the time

- pours, cuts with supervision, and mashes own food

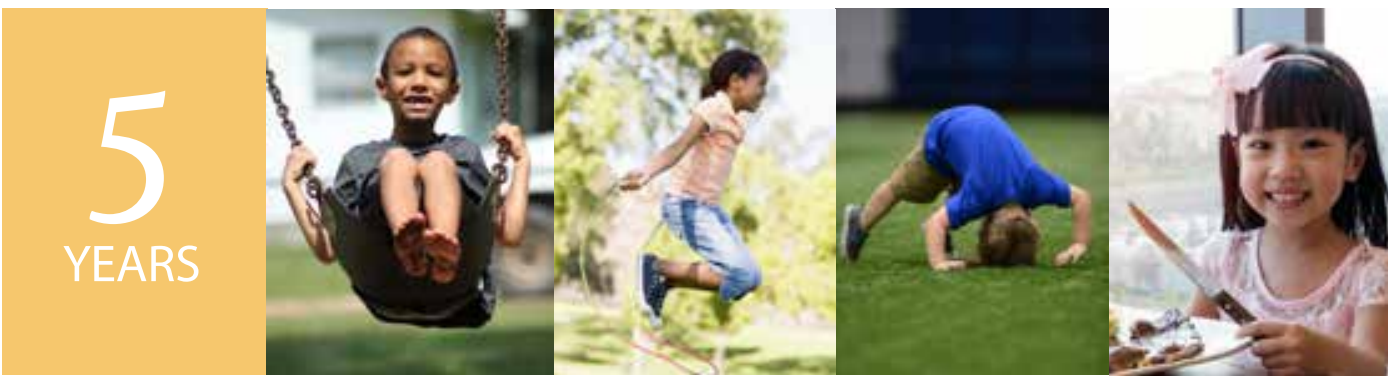

- swings and climbs

- can use a toilet alone

- stands on one foot for 10 seconds or longer

- hops and may be able to skip

- jumps rope

- can do a somersault

- uses a fork and spoon and sometimes a table knife 


\section{An Active Child in Our Community}

\section{0 minutes of moderate to vigorous physical activity}

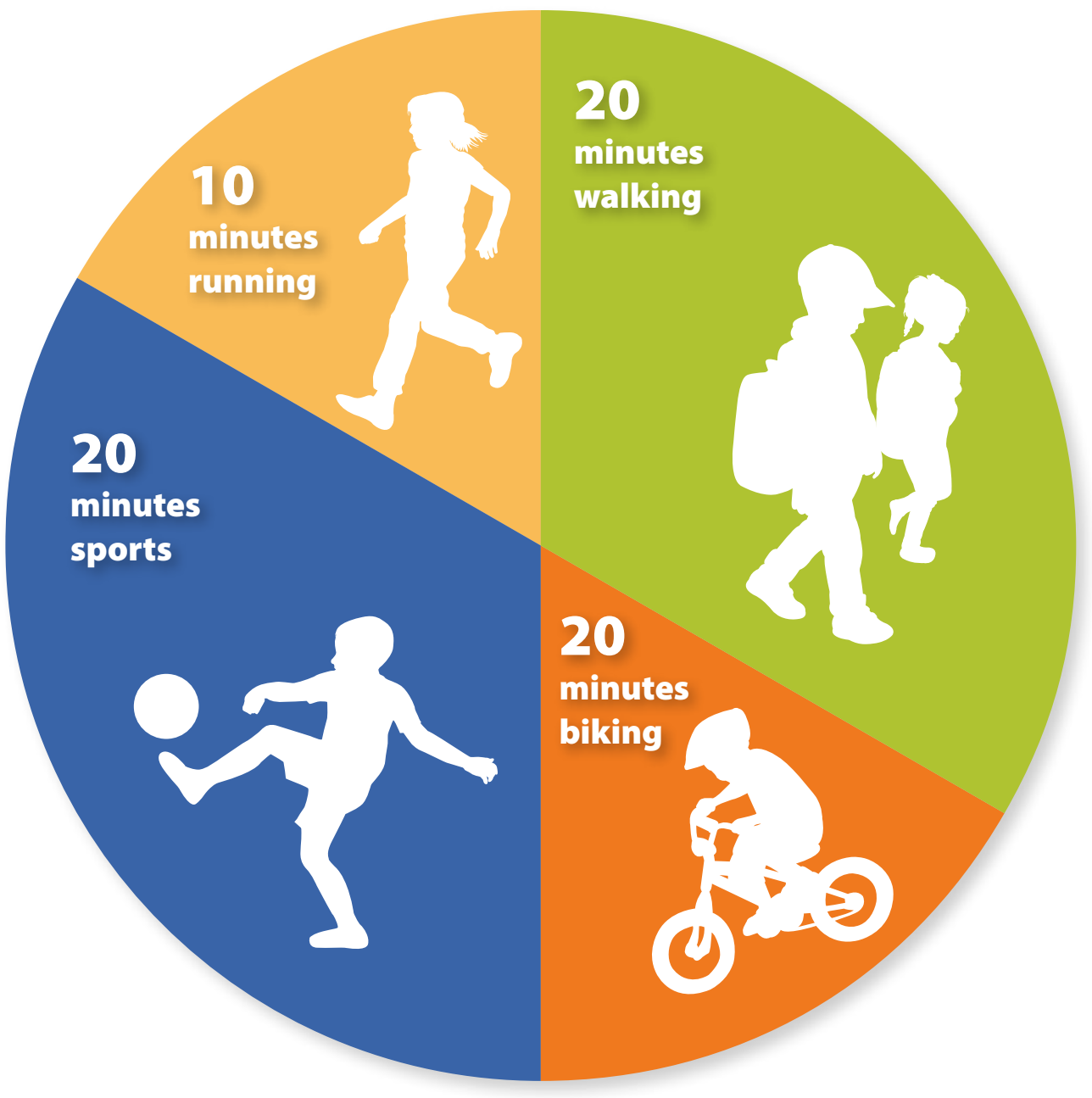




\section{A Less Active Child in Our Community}

\section{Less than 60 minutes of moderate to vigorous physical activity}

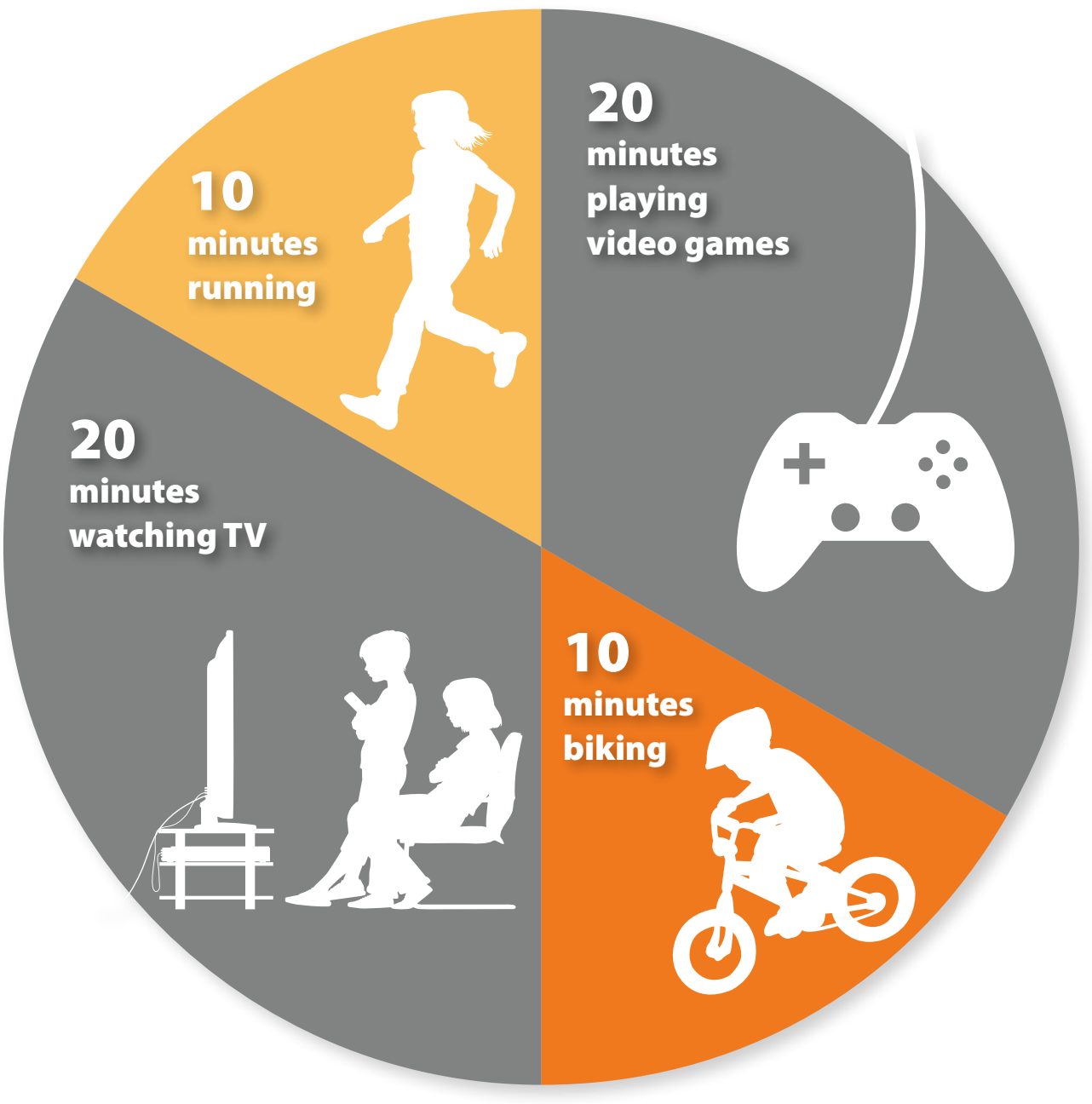


This material is based on work that is supported by the National Institute of Food and Agriculture, U.S. Department of Agriculture, under award number 2011-68001-30167 and Project CA-D*-NTR-2117-H.

\section{REFERENCES}

Food and Drug Administration (FDA). 2017a. How to read and use the nutrition facts label. FDA, gov website, http://www.fda.gov/Food/IngredientsPackagingLabeling/ LabelingNutrition/ucm274593.htm.

2017b. The new and improved nutrition facts label:

Key changes. FDA.gov website, https://www.fda.gov/ downloads/Food/IngredientsPackagingLabeling/ LabelingNutrition/UCM511646.pdf.

-2017c. The nutrition facts label: Look for it and use it (English). FDA.gov website, http://www.fda. gov/downloads/Food/IngredientsPackagingLabeling/ LabelingNutrition/UCM410486.pdf. . 2017d. Nutrition facts label: Look for it and use it (Spanish). FDA.gov website, http://www.fda.gov/ downloads/Food/IngredientsPackagingLabeling/ LabelingNutrition/UCM410487.pdf.

Ogden, C. L., M. D. Carroll, B. K. Kit, and K. M. Flegal. 2012. Prevalence of obesity and trends in body mass index among US children and adolescents, 1999-2010. JAMA: The Journal of the American Medical Association:40.

United States Department of Agriculture and U.S. Department of Human Health and Services. 2016. Dietary guidelines for Americans, 2015-2020. Health.gov website, https:// health.gov/dietaryguidelines/2015/.

— 2016. Appendix 1: Physical activity guidelines for Americans. Dietary Guidelines for Americans, 2015-2020. Health.gov website, http://health.gov/ dietaryguidelines/2015/guidelines/appendix1/\#table-a1-1,

University of Georgia Cooperative Extension Service (UGCES). 2002 Understanding food labels.

\section{FOR FURTHER INFORMATION}

To order or obtain ANR publications and other products, visit the ANR Communication Services online catalog at http://anrcatalog. ucanr.edu/ or phone 1-800-994-8849. You can also place orders by mail or FAX, or request a printed catalog of our products from

University of California

Agriculture and Natural Resources

Communication Services

2801 Second Street

Davis, CA 95618

Telephone 1-800-994-8849

E-mail: anrcatalog@ucanr.edu
(02019 The Regents of the University of California. This work is licensed under the Creative Commons Attribution-NonCommercial-NoDerivatives 4.0 International License. To view a copy of this license, visit http://creativecommons.org/licenses/by-ncnd/4.0/ or send a letter to Creative Commons, PO Box 1866, Mountain View, CA 94042, USA.

Publication 8585

ISBN-13: 978-1-60107-995-4

The University of California, Division of Agriculture and Natural Resources (UC ANR) prohibits discrimination against or harassment of any person in any of its programs or activities on the basis of race, color, national origin, religion, sex, gender, gender expression, gender identity, pregnancy (which includes pregnancy, childbirth, and medical conditions related to pregnancy or childbirth), physical or mental disability, medical condition (cancer-related or genetic characteristics), genetic information (including family medical history), ancestry, marital status, age, sexual orientation, citizenship, status as a protected veteran or service in the uniformed services (as defined by the Uniformed Services Employment and Reemployment Rights Act of 1994 [USERRA]), as well as state military and naval service.

UC ANR policy prohibits retaliation against any employee or person in any of its programs or activities for bringing a complaint of discrimination or harassment. UC ANR policy also prohibits retaliation against a person who assists someone with a complaint of discrimination or harassment, or participates in any manner in an investigation or resolution of a complaint of discrimination or harassment. Retaliation includes threats, intimidation, reprisals, and/or adverse actions related to any of its programs or activities.

UC ANR is an Equal Opportunity/Affirmative Action Employer. All qualified applicants will receive consideration for employment and/or participation in any of its programs or activities without regard to race, color, religion, sex, national origin, disability, age or protected veteran status.

University policy is intended to be consistent with the provisions of applicable State and Federal laws.

Inquiries regarding the University's equal employment opportunity policies may be directed to: Affirmative Action Contact and Title IX Officer, University of California, Agriculture and Natural Resources, 2801 Second Street, Davis, CA 95618, (530) 750-1397. Email: titleixdiscrimination@ucanr.edu. Website: http://ucanr. edu/sites/anrstaff/Diversity/Affirmative_Action/.
UCE REVIEWED reviewed for technical accuracy by University of California scientists and other qualified professionals. This review process was managed by ANR Associate Editor Katherine Soule.

web-11/19-LR/S

https://doi.org/10.3733/ucanr.8585 